\title{
From the Smart City to the Smart Community, Model and Architecture of a Real Project: SensorNet
}

\author{
Stefania Nanni, and Gianluca Mazzini
}

\begin{abstract}
- this article presents a conceptual, architectural and organizational model for the realization of a smart city based on a holistic paradigm as its cornerstone and on the new technologies as its enabling tools. The model is based on the concept of integration of the data belonging to different systems, through the development of a middleware, which allows the retrieval of data from various sources and their storage in a standard format in a new centralized database. The article also illustrates a real project concerning the integration of different sensor networks for the environmental monitoring that exemplifies and implements the main topics discussed. The issues related to its "governance" are also highlighted, not only from a strategic point of view, but also, and above all, from the perspective of its maintenance, which is an important and crucial feature for its "survival" over time.
\end{abstract}

Index terms - smart city, federal system, sensor networks integration, resources sharing.

\section{INTRODUCTION}

The definitions of the term "Smart City" have grown exponentially over the last few years, from those linking it primarily to the quality and use of services provided, regardless of the scope, the tools and technology used to produce them, to those identifying it, conversely, with the technology itself.

It's clear, though, that while it is no coincidence that this concept was born in the latest few years in which the development and the diffusion of new technologies have had tremendous acceleration, on the other hand it is equally true that the only intelligence of a technology is the one arising from the context and the purpose for which it is used.

The maturity of technologies for remote access to the services and the deployment of high-speed data transmission are enabling different users, organizations or private individuals to access all the available data and functions from anywhere and at any time, as if they were installed on their own PC.

Therefore it appears clear that the availability of an adequate connectivity, both in terms of performance and breadth, allowing access and exchange of information not only within a single system, but especially between different systems, is the prerequisite and the foundation of the "Smart City".

Manuscript received May 14, 2014; revised August 1, 2014.

Authors are with the LepidaSpA, V.le Aldo Moro 64, 40127 Bologna, Italy and University of Ferrara, Via Saragat 1, 44100 Ferrara, Italy.

E-mails: stefania.nanni@lepida.it,g.mazzini@ieee.org.
The definition that is here considered more appropriate for the term of "Smart City" and that is reflected in the model and in the example presented, is that of "Development of valueadded services, based on existing services, through the use of new technologies".

The concept of "smart city" will be here extended to the dimension of "smart city and community" (here summarized by the acronym SC\&C), which is composed by a set of subjects operating on the same territory and sharing resources and objectives, as they are the real beneficiaries and actors of the model presented hereafter.

\section{THE STATE OF THE ART}

The integration of data belonging to different and not interoperable systems is a problem encompassing multiple application environments, and presenting several analogies both from the opportunities and the implementation criticalities points of view.

For what it is concerning the environmental monitoring systems, the majority of the existing sensor networks is based on proprietary technologies and protocols, usually wireless, which makes them coherent and performing on one hand, but also closed and not interoperable [1].

In the first phase of the project here described, a model of integration was considered, based on the introduction of a "black box" capable of interfacing to both proprietary controllers and proprietary sensors, and being the element of standardization in terms of both protocol and transmission network of the different sensor networks [2]. The implementation of this type of solution has faced several issues, very often more organizational than technical, because of the need of cooperation from the vendors of the various parts of the monitoring systems.

Examples of sensor networks integrations do exist at transmission layer level [3] (overlay networks), but limited to homogeneous technological scopes. And regardless of whether they are wireless [4], IP [5],[6] or internet based [7], they all share the assumption of nodes having a non negligible computational capacity, allowing them to run multiple applications at a time, at least the native one and those which are needed for the integration.

Some examples of platforms integrating sensors of heterogeneous technologies are currently available, but they are usually based on a distributed architecture of data collecting nodes (servers), and typically oriented to monitoring individual phenomena such as river environment [8], landslide detection [9] or air pollution level [10]. 
In recent times, examples of "social" shared platforms are becoming more and more popular, having their main feature in the use of smart phones as sensors and drivers for capturing data of diverse kinds [11]. Their approach is dramatically different from the classical monitoring methodology, as they oppose to accurate and well localized measurements their intensive use of statistical analysis applied to a wide, but not necessarily accurate, set of samples. Such examples are currently limited to circumscribed application scopes, such as sport activity tracking, traffic monitoring, etc. [12], mainly depending on the kind of sensors available on the smart phones or portable (or even wearable) electronics, but they are still far from being the answer to the need of integrating the large number of sensors networks already installed with their respective logistics and application constraints.

The solution proposed here is different from all those previously described as it allows the merge of every kind of environmental monitoring, overcoming the limitation to a single natural phenomenon, it is independent from the transmission technology used, as it is does not put any constraint on technology and performances on the nodes of the merged sensor networks, and it is independent from the sensors networks suppliers, because it carries the burden of developing the necessary interfaces to implement data retrieval, whatever the technology, the communication protocol or the level at which data are made available (database, text file, control unit, etc.).

The project presented has been developed using open source software, but the same architecture can also be achieved with equivalent cost and performance through commercial products [13], [14], [15], leaving the choice of the most suitable solution to corporate policies criteria rather than to technical or functional issues.

\section{SMART CITY MODEL}

\section{A. Conceptual model}

The diversification of the needs, objectives and roles of the organizations operating in a given metropolitan area must be able to take advantage of the independence that a neutral approach towards the different available technologies can offer and of all the opportunities related to that.

The "Smart City", considered in the aforementioned context, can be imagined as a "smart integration of its coexisting subsystems", each one having its own original and primary purpose, different from all the others, and for this reason being self consistent and self-sustaining.

This concept outlines an ontological model of "smart services" as "federated services" and especially emphasizes the precondition for their maintainability over time, which is the most critical aspect of any system and therefore also of those in object [16].

The existence of a purpose, an interest and an organizationspecific reference for each subsystem ensures, as a matter of facts, its maintenance over time.

The "federated" approach in place of the "unitary" one, represents a model which is really viable and sustainable. It's also the only one allowing the intelligent (smart, in fact) reuse of existing systems originated in an independent manner and hence possibly not interoperable [17]. The federal model involves by its specific nature the development of appropriate specific interfaces enabling the virtualization and the integration of each component subsystem, regardless of the platform and the technologies any of them are made with. This aspect is crucial in order to protect and enhance the investments already incurred for the existing systems.

Figure 1 highlights the possible exchange of information among different systems in the area, thus making each one of them more effective and also making more effective the overall result itself.

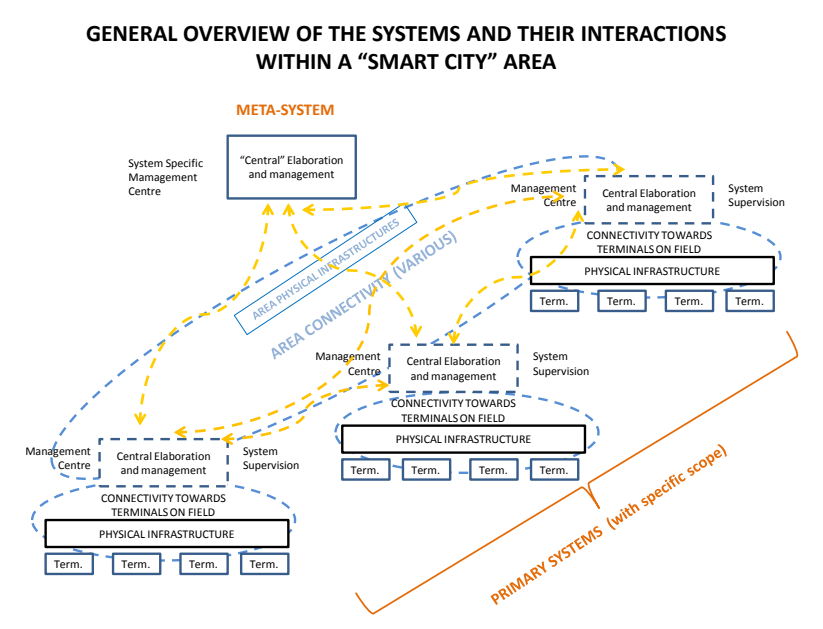

Figure 1 - Possible exchange of information among systems

In addition to the added value achieved through the capability of accessing their content by systems which are "smart" in the sense just described, an additional added value should also be stressed, produced by the savings that can be achieved through the synergic and coordinated use of two elements which are necessarily part of the individual subsystems: i.e. connectivity and infrastructure.

The connectivity exploited by each subsystem may be partly dedicated to its own purpose, but in part it may also be shared with other systems. In general, the part of "shared" connectivity may be supplied by public telecom operators (fixed and mobile), or may be a result of a specific connectivity mix implemented for various purposes (subnets in Wi-Fi technology, dedicated optical fibers, etc.).

The same sharing potential also applies to the physical infrastructure. In fact infrastructure is generally one of the most expensive factors in any system. Sharing it in terms of physical media, power supply availability or even physical equipment can obviously be an added value to the individual subsystems [16].

Even in this case, allocating to each subsystem the maintenance and the operation of its own resources is the best assurance of efficiency and continuity of their specific basic services, and, as a consequence, that of the value-added ones.

This framework leaves anyway open the possibility for transferring to a common central organization the maintenance of those resources used by more than one system, as well as 
the adoption of "sharing" models, borrowed, for example, from the long experience of the cellular networks.

It is important to highlight that while the approach of preserving individual subsystems is a principle of general validity whose benefits have been widely discussed, the assumption that makes the added-value services feasible is the concept that the mission of the "federative" system is to create the virtualization layer allowing to integrate the individual subsystems as required.

The issue of standardization, which would greatly facilitate interoperability and interactions among systems, is very complex. The related requirements of forecasting and beforehand planning are everything but easy. In any case it falls outside the scope of this paper.

It has to be mentioned here that the use of SOAP architectures for federating different services is a well established solution and is theoretically also applicable within the model that has been described [6]. The distinctive aspect that is highlighted here is that in order to create value added services based upon self-sustaining subsystems already in place, the development of integration interfaces has to be undertaken, by the federating entity which is at a time a natural reference, the responsible and the promoter for the new services.

As a general consideration it can thus be said that until standards will be established and adopted on a large scale, the model here presented offers a viable and valid solution for all those areas, which are the most commonly encountered, in which diversification and their own peculiarities should not be seen as obstacles, but as an opportunity to exploit [18].

\section{B. Architectural model}

The architecture of the "federal" model aims at providing a solution collecting and normalizing data originated from various systems, ensuring to both the supplier and the system integrator the maximum freedom in managing and structuring the data.

The proposed architecture provides an interconnection middleware between the different data sources and the central system, acting as a data collector from those different sources and as a data normalizer facing the central system, as shown in Figure 2.

The architecture consists of a series of atomic modules, for data retrieval from individual sources, and of their manager which oversees their activation and coordination.

Each module contains the access rules and the required commands for retrieving data from a specific source or database and for storing them in a standard format on a centralized database.

In order to acquire data from heterogeneous sources and use them in a contextual and correlated mode a standardization process is necessary.

The creation of a standardized data stream is one of the added values offered by the "federator" system, which transforms the data from the different sources into a single standard format, regardless of the technologies, the interfaces and the formats of the original subsystems.

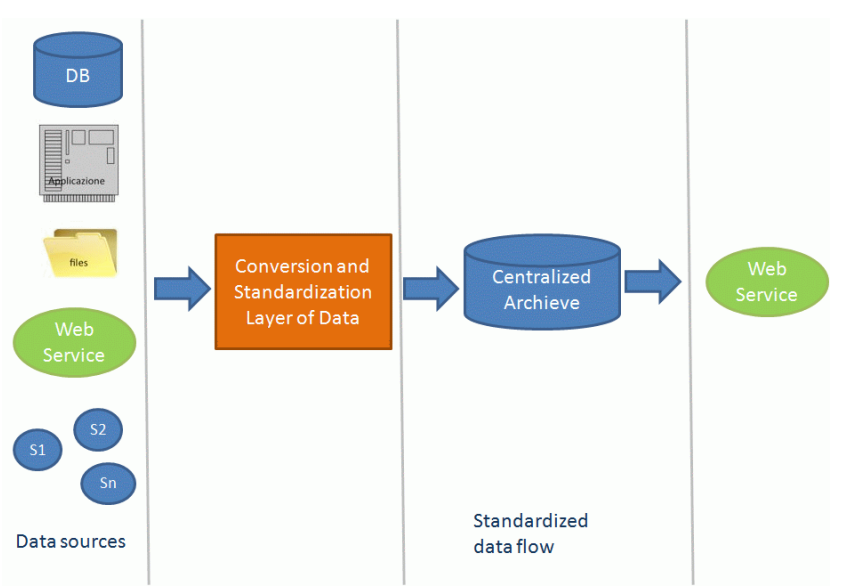

Figure 2- Flow of collection, standardization, storage and access of the data

Each sub-supplier, but also systems or third parties will be granted access through the appropriate standard interfaces to the standardized real-time data flow and to its historical base.

The creation of a central repository, gathering and storing all available data in a standard format is a solution having its natural application in all those cases, as the one in this article, in which the collected data have a permanent validity and are not subject to change or updates. In these cases, in fact, the data storage in a centralized repository allows to run once and forever the process of data collection and standardization and to increase the reliability because it eliminates the dependence on the availability of the overall data from that of the individual subsystems, at least for what is concerning the historical data base.

Vice versa, a strictly federal model as opposed to a centralized archive, is a system of automatic data collection and standardization from different sources which may be applied in all those scenarios, such as e.g. the ones relating to the "Cadastre of infrastructure", in which the original information is subject to change [17]. In fact in these cases the assumption that the original subsystems will remain the only holders of data is the fundamental solution to the problem of their update in the central system.

\section{Functional model}

The main function of the "federator" system is to collect and normalize data from various available sources and make them available in a standard format to all the interested systems or third parties.

The "federator" system can in general also be equipped with a data analysis layer and an interactive interface which, suitably profiled, grants data access to all interested users. In other words, as shown in Figure 3, the "federator" system can be equipped with some basic functionality for management and display of the integrated data, which constitutes a first important and significant output of the new overlaid system.

These auxiliary functionalities can be useful mostly for those who do not have a platform of their own for storage, access and analysis, but may be also of use for those who, while already having a platform for storage and data management, are in need of implementing a new specific monitoring 
function (or functions) and can therefore focus on the installation and maintenance of the new devices in the field.

Sharing a common data management platform, instead of replicating them, has the advantage of cost reduction and simplification of platform management and maintenance processes.

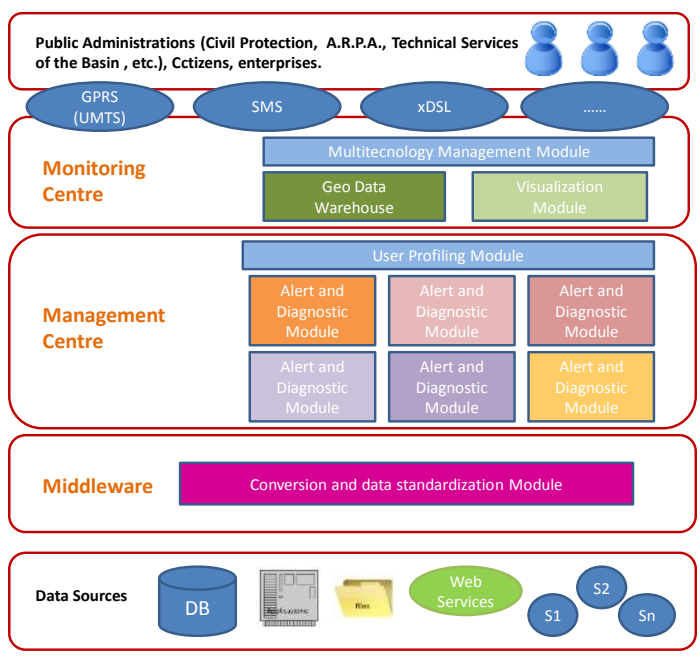

Figure 3-Block diagram of general architecture inclusive of auxiliary functionality

\section{Management issues related to the model}

In a "smart system", as it is intended in this article, it is important to analyze the problems of operation and maintenance of the "federal" system.

It has already been highlighted that the existence of an objective specific to each primary subsystem is granting that all their maintenance problems will be addressed with the necessary efficiency and continuity by the organizational entities accountable for their functionality.

The interaction among the primary subsystems will then be subject to clear responsibilities and formalized SLA within the "federal system".

This approach ensures the coverage of most of the "survival" needs of the "federal" system, but doesn't include them all. Some areas still remains not covered, such as the ones related to the connectivity and shared infrastructures.

\section{D.1 Connectivity}

As it has already been pointed out, the availability of an adequate connectivity at low cost is an essential assumption for the whole concept of SC\&C, for what is concerning the individual subsystems, but also and especially for what is concerning their interaction.

If the impact of the shared part of connectivity between the systems is important, but none of the primary systems uses it in a prevalent manner, it is natural to think to concentrate its management in a single organization throughout the SC\&C, to promote the optimization of resources, costs and skills.

\section{D.2 Civil infrastructures}

This category includes public lighting, traffic signal systems, totems of the parking fee, underground infrastructure (ducts and wells), etc. Each one of these infrastructures has an intrinsic value and carries a potential opportunity of reuse, because of its presence in the territory (including the permit of its construction) and, in general, by the presence of its own source of energy supply.

The sharing of such kind of resources among different users is not a new problem. The "site sharing", in the case of the cellular networks, has become increasingly widespread. In general, the most common pattern is that a given subject, who is the owner of the resource, "rent" it to third parties.

A similar scheme could also be used in the case of a SC\&C area, not always and not necessarily with passage of economic values (that would have little sense, for example, in the case of Public Administrations), but with the formalization of a "service trading", which include, for example, the maintenance of the physical media and the assurance over time of the capacity and continuity of power supply as required.

\section{D.3 Data center}

Another category of infrastructures is represented by the physical sites hosting the "servers" that are part of every primary subsystem. It's obvious that an approach using "cloud" technology within a regional or metropolitan area provides advantages in efficiency, cost and safety.

\section{D.4 Operational centers}

A third category, similar to the previous, is composed of sites hosting the so-called "operational centers", i.e. those centers generally used for security and territory management purposes. A very extended metropolitan or a regional area, which represents a unique SC\&C environment, could include several operational centers. Also in this case it is reasonable to think of a "federation" approach, that aggregates similar installations in one place, enhancing scale factors benefits, while keeping distinct and independent management for each organization.

\section{E. Governance model}

For what is concerning the problem of "governance" of a SC\&C environment, there is no doubt that an important part of "governance" must necessarily be centralized, due to the fact that the "holistic" approach is a basic characteristic of SC\&C domains, and it can only be managed in this way.

Only in this way opportunities can be identified for interaction between systems, for creation of "metasystem" and, in general, for identification and promotion of exploitation synergies.

The problem is to determine what is the best organizational solution to resolve this issue.

When analyzing the governance model, it would therefore seem very reasonable that a "federal" approach, in which only a part is "centralized", should harmoniously co-exist with the 
management of the individual subsystems in a "federated" manner.

The "federal" part should declare and make available the data and information owned by each subsystem.

The "centralized" part, being the natural aggregator for the "federated" one, should also be the place in which the overall strategy of SC\&C resides, or at least the preferred place to identify and to verify priorities and opportunities of certain individual initiatives, or to supervise the agreements that necessarily follow the same realization of synergies .

It should also be the place where the information security problems, potentially accentuated by increased distribution and greater access to the data, are addressed.

The organizational way to achieve all this (by department, agency, in-house company or other mechanism) can only depend on the individual local situations and partly from previous evolution of the context.

It should finally be pointed out the contribution that this centralized entity could take in the preparation of the SC\&C environment, supporting the management of major emergencies (not of course the daily ones, for which the individual organizations are accountable).

Emergency management is indeed the case where the greatest possible integration (and correlation) of information, coming from different areas, becomes particularly important. The application example, which will be presented below, is a clear demonstration of this last and very important aspect and, more generally, of the entire model illustrated.

\section{ADVANTAGES OF THE MODEL}

The advantages of the model presented can be summarized in the following points:

1. Preservation and enhancement of the investments made on existing systems;

2. Protection of the technical, technological and organizational autonomy of the individual subsystems and of their owning entity;

3. Creation of a single centralized repository from which to draw all available data in a standard format;

4. Definition of a single data collector for every individual subsystems;

5. Hierarchically expandable architecture;

6. Architectural model which can be adopted in different scenarios of application;

7. Operational and organizational model for the realization of "smart cities".

The majority of the above listed advantages have been either described inside the present document or are self-explanatory.

Nevertheless the fourth item in the above list requires some more detailed argumentation: in a general trend pushing more and more towards data sharing, the fact of creating a single reference entity to whom to route potential changes or updates on the interfaces or on available data, is a remarkable simplification granting superior reliability related to maintenance or management of the whole "ecosystem".

The users themselves, accessing to a single interface for data retrieval, are unaffected by technical or technological evolutions of the individual subsystems which are managed instead at "federator" system level.

The case of environmental monitoring described below is a typical and significant case of use of the previously illustrated model and of its consequent advantages.

\section{AN EXAMPLE OF ACTUAL APPLICATION OF THE MODEL: "SENSORNET"}

\section{A. The Project}

SensorNet is a project implemented within the so-called "Telematic Regional Planning Framework 2011-2013" of the region of Emilia-Romagna. Its main purpose is that to promote data sharing and the rationalized and optimized use of infrastructure and connectivity.

The territory of the Emilia-Romagna Region is currently populated by thousands of sensors belonging to different monitoring systems that are not interoperable and not shared, which have been developed in different times with different technologies, owned by different public entities (Arpa, Technical Services of the Basin, Municipalities, Region, etc..) and responding to different needs and monitoring purposes (pollution, traffic, landslides, etc.).

This plethora of devices are often duplicated in the functions and positions and almost always exploited in raw, partial and closed scenarios.

The means used for data collection are also very variable, ranging from manual to automatic data collecting, through different telecommunications networks and technologies (ADSL, fiber optic, GSM, GPRS), all of this with costs and management problems that may be critical if not even disabling in a general perspective.

"Federating" all these sensors through the creation of a "federator" system will create synergies allowing to minimize duplication of sensors and sites, and to take advantage of the opportunity presented by the existing mobile network denominated ERRETRE and mainly used for emergency purposes, for collecting data and taking also advantage of the existing broadband network called Lepida (reserved to public entities within the region) for sharing them. Implementing such a federation architecture is not only possible and useful but also necessary, if not mandatory [19].

The SensorNet platform has been implemented and is now integrating about a thousand different sensors managed by different entities, i.e. mainly coming from the rain monitoring system of Arpa, from the Regional Traffic Monitoring System (MTS) and from the landslide monitoring of Technical Services of the Basin [20].

The table below contains the details of the sensors integrated within the system up to now, as shown in Figure 4.

\begin{tabular}{|l|r|l|}
\hline Kind of sensors & Number & Owner \\
\hline inductive coils & 591 & Emilia-Romagna Region \\
\hline rain gauges & 238 & Arpa Emilia-Romagna \\
\hline hydrometers & 197 & Arpa Emilia-Romagna \\
\hline temperature sensors & 174 & Arpa Emilia-Romagna \\
\hline inclinometers & 8 & Technical Services of the Basin \\
\hline
\end{tabular}




\begin{tabular}{|l|r|l|} 
piezometers & 3 & Technical Services of the Basin \\
\hline prisms & 144 & University of Modena \\
\hline sound level meters & 2 & LepidaSpA \\
\hline manual inclinometers & 156 & TechnicalService of the Basin \\
\hline strain gauges & 5 & Fanano Municipality \\
\hline
\end{tabular}

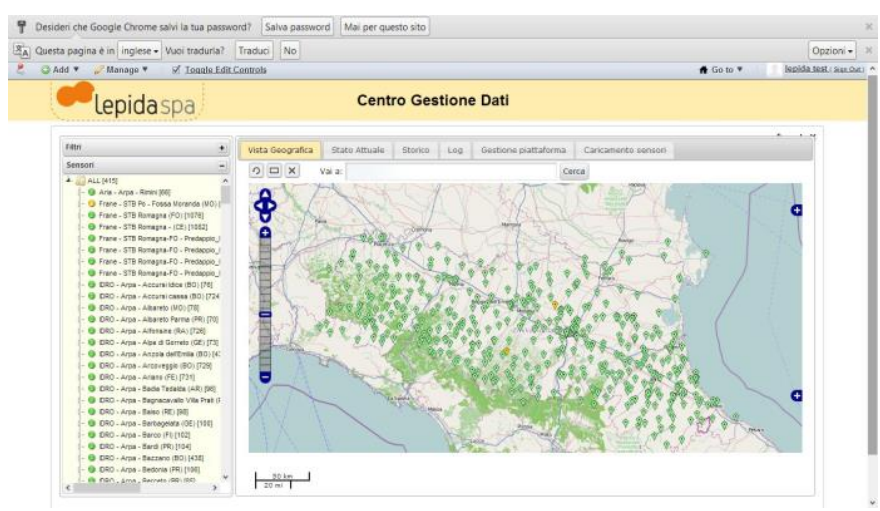

Figure 4- Main web interface of SensorNet platform

\section{B. Use of SensorNet in a more effective early warning scenario}

The term early warning (EW) indicates alarms that arise in the time interval between the moment in which phenomena potentially triggering a dangerous event are observed and the time at which the event happens. Time scales characteristic of early warning are different for different types of events:

- from seconds to tens of seconds for earthquakes;

- from minutes to hours for tsunamis;

- from hours to days for weather events;

- from hours to days to floods and landslides;

- from hours to weeks to volcanic eruptions.

The adoption of early warning (EW) methodologies is considered to be essential to cope with disasters (not just natural) in a world where the population is not only increasing, but it is concentrated in megacities of several or tens of millions inhabitants. In fact, the EW appears as a keyword in all documents addressing the problem of risk reduction, both nationally and internationally.

Italy is a country with a high landslide and flooding risk. In Emilia- Romagna monitoring and control of the territory is delegated to several agencies, such as Arpa and Technical Services of the Basin (STB), which are dealing with the monitoring of different environmental phenomena, such as the rainfall and river levels on one hand and landslides on the other.

As previously mentioned, currently each agency has its own independent and non-interoperable monitoring system, which makes it difficult, if not impossible, to share data.

Through the SensorNet platform it has been made possible to integrate real-time data from various subsystems and to make them available in the same context. This result is essential for the joint analysis and correlation of data coming from different systems.

With specific regard to the study of landslides, the integration on the same platform of the data belonging to the
Arpa rain monitoring system and to the Technical Services of the Basin landslide monitoring system, allows the study of the relationship between triggering factors, such as the rain, and the movements of landslides, as shown in Figure 5.

Through the continuous and contextual monitoring of a range of available parameters and the real-time analysis and mutual correlation of their dynamics it is possible to identify conditions indicating the generation of potentially dangerous events.

The SensorNet platform constitutes therefore a fundamental support for the analysis of risk and alert situations related to landslides, and a formidable governance instrument available to those subjects, such as the Civil Defence Department, which are institutionally in charge of predicting and preventing risks over the territory.

The system is developed and managed by LepidaSpA, which is the in-house ICT company of the Emilia-Romagna region and of all the public administrations of the territory. Thanks to its central, impartial and referenced role, LepidaSpA is one of the preferred subjects to provide value-added services to the community consisting of all public administrations of the Emilia- Romagna, on top of its role of managing the regional optical fiber telecommunication network (Lepida).

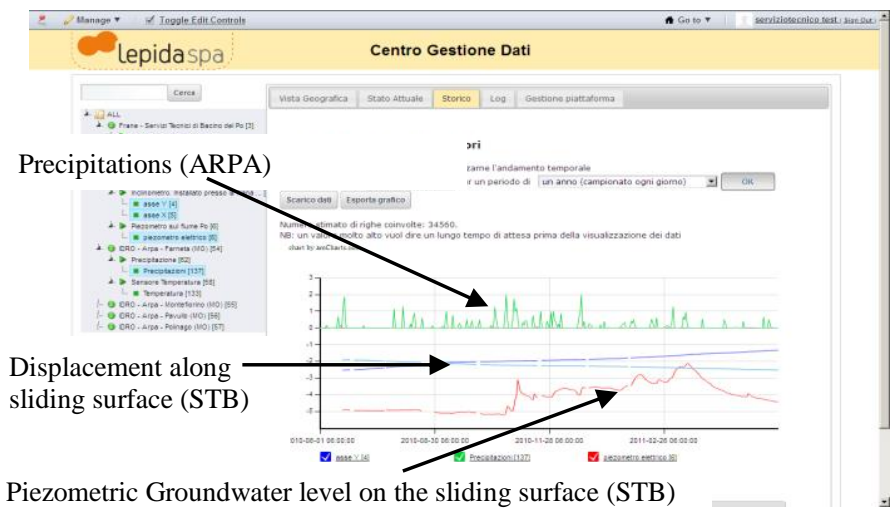

Figure 5-Aggregation of data from different data sources: $\underline{S T B}$ e ARPA

\section{CONCLUSION}

The need and the subsequent opportunity of integrating the information coming from different monitoring systems is applicable and is common to many areas. The definition of standards that promote interoperability is certainly a desirable goal, but it does not address by itself all the opportunities that a more flexible approach can produce more quickly and with more reliable results.

The model for creating a virtualization layer that allows to standardize all what has not been originally standardized is considered as a pragmatic approach, allowing also the creation of value-added services in a relative fast time-frame.

The model of data sharing follows a process that is neutral from a technical and organizational standpoint, is more immediate than what it takes to implement the sharing of infrastructure and connectivity.

It therefore represents a first comparatively easy step towards a new concept of "Smart City", obviously linked to the quality 
and the utility of the services that it is able to offer, but also to the models which appear most suitable from the point of view of the optimization of the necessary resources and adequate manage and maintain them as a whole in time.

The presented model has ultimately highlighted that the basis for a smart city is a smart community, finding in the Public Administrations, according to the characteristics described in this paper, the most meaningful and widespread examples.

\section{REFERENCES}

[1] I. F. Akyildiz, W. Su, Y. Sankarasubramaniam, E. Cayirci: "Wireless sensor networks: a survey", Computer Networks, Volume 38, Issue 4, 15 March 2002, Pages 393-422.

[2] Elisa Benetti, Chiara Taddia and Gianluca Mazzini: "Environmental Monitoring Supported by the Regional Network Infrastructures", Environmental Monitoring, Dr Ema Ekundayo (Ed.), ISBN: 978-953-307-724-6, InTech, DOI: 10.5772/27130.

[3] Luis Garcés-Erice, Daniel Bauer, Paolo Scotton: A flexible and scalable message broker for sensor network integration, COMSWARE '09 Proceedings of the Fourth International ICST Conference on COMmunication System softWAre and middleware, No.4.

[4] Christos Efstratiou: Challenges in Supporting Federation of Sensor Networks, Position paper in NSF/FIRE Workshop on Federating Computing Resources, Princeton, NJ, May 11-12, 2010.

[5] Dogan Yazar, Adam Dunkels: Efficient Application Integration in IP-Based Sensor Networks, BuildSys '09 Proceedings of the First ACM Workshop on Embedded Sensing Systems for Energy-Efficiency in Buildings, Pages 43-48.

[6] Akbar Ghobakhlou, Alexander Kmoch, Philip Sallis: Integration of Wireless Sensor Network and Web Services, 20th International Congress on Modelling and Simulation, Adelaide, Australia, 1-6 December 2013.

[7] Jeff Shneidman, Peter Pietzuch, Jonathan Ledlie, Mema Roussopoulos, Margo Seltzer, Matt Welsh: Hourglass: An Infrastructure for Connecting Sensor Networks and Application, Harvard Technical Report TR-21-04.

[8] Kolar, Harry R., Cronin, John, Hartswick, Perry, Sanderson, Arthur C., Bonner, James S., Hotaling, Liesl, Ambrosio, Ron F., Liu, Zhen, Passow, Michael L. and Reath, Mark L.: Complex real-time environmental monitoring of the Hudson River and estuary system, IBM Journal of Research and Development 53, no. 3 (2009): 4.

[9] Maneesha V. Ramesh, Sangeeth Kumar, and P. Venkat Rangan: Wireless Sensor Network for Landslide Detection, SENSORCOMM '09. Third International Conference on Sensor Technologies and Applications, 2009.

[10] http://www.ec.gc.ca/rs-mn/

[11] John G. Breslin, Stefan Decker, Manfred Hauswirth, Gearoid Hynes, Danh Le Phuoc, Alexandre Passant, Axel Polleres, Cornelius Rabsch, Vinny Reynolds: Integrating Social Networks and Sensor Networks, W3C Workshop on the Future of Social Networking, 15-16 January 2009, Barcelona.

[12] ] http://www.sense-os.nl/

[13] http://www-01.ibm.com/software/data/integration/

[14] http://www.oracle.com/us/industries/public-sector/intelligencehib-alerts-br-1536069.pdf

[15] http://www.abodata.com/abodata/images/stories/Brochure/aboda ta_iwpc\%20-\%20platone\%20and\%20m2m_v3.pdf

[16] Agenzia per 1'Italia digitale, Architettura per le comunità intelligenti: visione concettuale e raccomandazioni alle Pubbliche Amministrazioni, Ottobre 2012.
[17] S. Nanni, G. Mazzini, A Federal Register For Telecomminications Infrastructure, Proceeding of Workshop on ICT, SoftCOM, September 2012.

[18] Jung-Hoon Lee, Marguerite Gong Hancock: Toward a framework for Smart Cities: A Comparison of Seoul, San Francisco \& Amsterdam.

[19] C. Taddia, S. Nanni, and G. Mazzini: Technology Integration for the Services Offered by the Public Administrations, IARIA Neutral 2009, August, 23-29, Cannes, France.

[20] sensornet.lepida.it

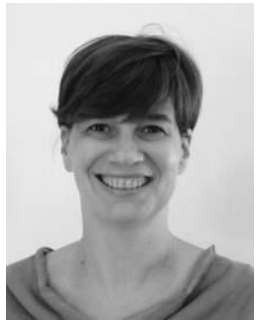

Stefania Nanni received the M.S. degree in electronics engineering (summa con laude) from the University of Bologna, Italy, in 1992. From 1992 to 2009 she worked as a system engineer for an Italian firm leader in emergency lighting, domotics and alert systems.

Since 2009 she is working in the R\&D department of LepidaSpA, Bologna, Italy where she is in charge of the ICT Laboratory for Public Administrations. She is involved in all the aspects concerning the deployment of Smart Cities paradigm through the prototyping of innovative ICT platforms, providing added value and new services for the P.A. in the Region of Emilia Romagna, with particular focus on the issues related to integration of data belonging to different systems in the fields of sensor networks and cadastral systems.

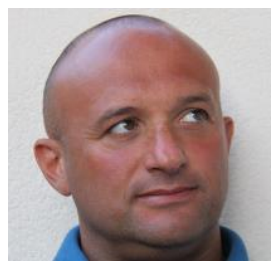

Gianluca Mazzini was born in Bologna in 1968. In 1992 he graduated in Electronic Engineering (summa cum laude) and in 1996 he got the Ph.D. degree in Electrical Engineering and Computer Science at the University of Bologna. In 1996 he joined the University of Ferrara in the role of Assistant Professor and in 2002 he held the position of Associate Professor. He is a Senior Member of the IEEE. The research carried out since 1993 are related to: spread spectrum communications; applications of chaos to telecommunications; architectures for efficient radio local area networks, cellular and ambient; routing strategies in mobility sensor networks; capacity in telecommunications system; peer-topeer networks; networks with multimedia traffic; information security. He is author or coauthor of more than 250 international publications in books, journals or conference proceedings. Google Scholar in November 2012 reports over 4700 citations with an h factor of 37 and an i10 factor of 58 . The teaching shows more than 50 editions of university courses in 12 different categories. He has been the supervisor of over 140 theses and tutor for $14 \mathrm{PhD}$ students. He has been co-organizer of two international conferences, guest editor of the Proceedings of the IEEE, has served as Associate Editor for the IEEE journals for 9 years, has served as TPC member for more than 40 international conferences. He has had roles in coordinating over a dozen of projects with an international or national level, including four European projects. As first researcher in role for TLC in University of Ferrara, he founded the research group in TLC area and has established a structured series of collaborations with other organizations, including: ARCES at the University of Bologna, IEIIT at the CNR, CNIT. He has been a member of seven scientific committees and 7 boards of directors or management. He was CEO of LepidaSpA. 